\title{
Infra-vesical Obstructive Uropathy Secondary to Benign Prostatic Enlargement.
}

\begin{abstract}
a Department of Radiology, Mansoura Health Insurance Hospital, Egypt. ${ }^{\mathrm{b}}$ Department of Urology, Faculty of Medicine, Urology and nephrology center, Mansoura University, Egypt.

Correspondence to: Ghada M. Abdel-Rafee, department of Radiology, Mansoura Health Insurance Hospital, Egypt,
\end{abstract}

Ghada M. Abdel-Rafee ${ }^{\mathrm{a}}$, Hassan Aboul-Enein ${ }^{\mathrm{b}}$

Email:

doctorghada2016@yahoo.com

Received: 2 May 2020

Accepted: 4 October 2020

\begin{abstract}
:
Background: Urinary tract obstruction is a common clinical problem. The obstruction of the urinary flow may be acute or chronic, partial or complete, unilateral or bilateral, and may occur at any site of the urinary tract. Aim: Imaging of complication of obstructive uropathy below the level of urinary bladder in adult male patient with enlarged prostate. We put urethral catheter to relief symptoms. Serum creatinine, PSA \& urine analysis were within normal \& the general condition was stabilized. Methods: Bedside ultrasound is used to scan the upper \& lower urinary tract. We report a case of a male patient aged 50 y old, diabetic \& hypertensive $\&$ complained of sudden onset of bilateral flank pain \& urine retention. It is preceded by LUTS including: frequency, nocturia, dysuria \& incomplete evacuation of bladder since 1 month. No previous surgical operation. No family history of renal stones. Results: Ultrasound is a valuable diagnostic tool in obstructive uropathy. Conclusion: Scan of pre-voiding \& post-
\end{abstract} voiding renal tract is very important to realize the etiology \& the level of urinary tract obstruction.

Keywords: Infra-vesical, obstructive uropathy, prostatic enlargement.

\section{Introduction}

Obstructive uropathy is when the urine can't

flow through ureter, bladder or urethra due to some type of obstruction. Instead of flowing from kidneys to bladder, urine flows backward or refluxes into kidneys causing swelling \& damaging them. It sometimes 
leading to renal dysfunction (obstructive nephropathy). Symptoms, less likely in chronic obstruction, may include pain radiating to the T11 to T12 dermatomes and abnormal voiding (e.g., difficulty voiding, anuria, nocturia and/or polyuria). Diagnosis is based on results of bladder catheterization, cystourethroscopy, and imaging (e.g., ultrasonography, CT, pyelography), depending on the level of obstruction. Treatment, depending on cause, may require prompt drainage, instrumentation, surgery (e.g., endoscopy, lithotripsy), hormonal therapy, or a combination of these modalities. Pathologic findings consist of dilation of the collecting ducts and distal tubules and chronic tubular atrophy with little glomerular damage. Dilation takes 3 days from the onset of obstructive uropathy to develop; before then, the collecting system is relatively noncompliant and less likely to dilate. The calculi are most common in young adults, while prostatic hyperplasia or carcinoma, retroperitoneal or pelvic neoplasms, and calculi are the primary causes in old age. (1)

\section{Subject \& methods}

A case report study of a male patient aged $50 \mathrm{y}$ with change in urinary habits. It was done in Radiology department of Mansoura
University Hospital, Mansoura, at 25 to 30 April, 2020 time range.

Written informed patient consent for publication was obtained. He had bilateral flank pain \& difficulty passing urine, slow stream, dribbling, nocturia, incomplete evacuation of bladder, decrease urine output $\&$ hematuria since 2 days. We had to convey to the requesting physician in case of acute urine retention.

\section{Results}

Pre-voiding scan: Bilateral grade II hydronephrosis \& hydroureter. Enlarged prostatic volume $(75 \mathrm{cc})$, homogeneous echo pattern of prostate, grade III intra-vesical protrusion of the medial lobe in the bladder base $(20 \mathrm{~mm})$. No associate focal lesions, masses or foci of calcification. Over distended bladder with diffuse circumferential mucosal thickening of the bladder with fine internal echoes inside. Post-voiding scan: Bilateral grade I hydronephrosis \& hydroureter. Post-voiding residual (PVR) is $(220$ cc). Bedside ultrasound is necessary in such case to scan the upper \& lower urinary tract. Imaging tools can point out blockage to your doctor $\&$ so take the appropriate intervention (2 \&3) 


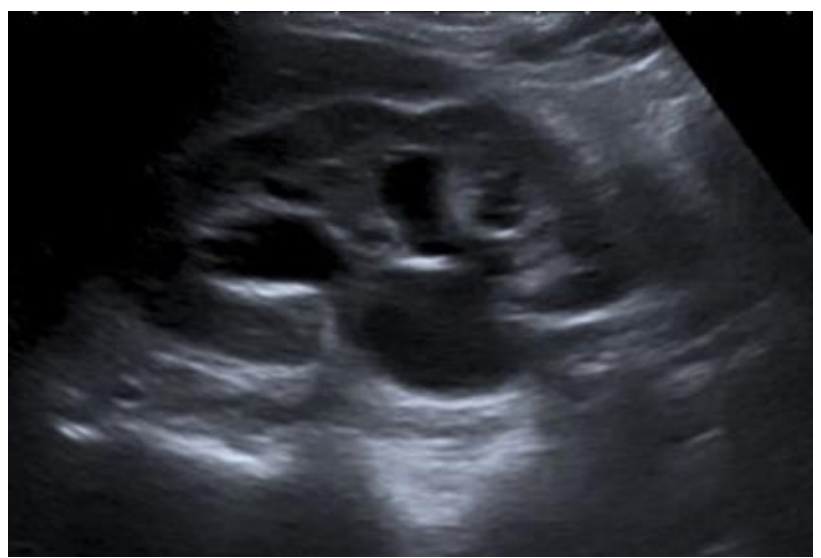

Figure (1): Abdominal ultrasound, pre-voiding scan revealed grade II hydronephrosis \& hydroureter. No stones or masses.

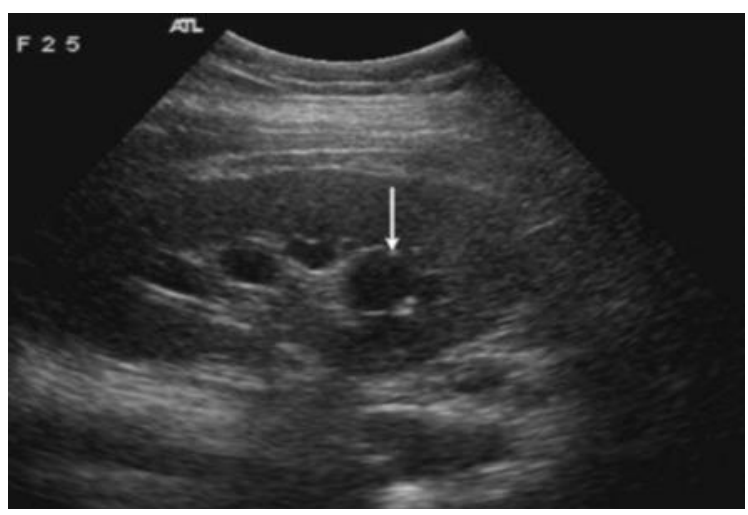

Figure (2): Abdominal ultrasound, post-voiding scan revealed grade I hydronephrosis \& hydroureter. No stones or masses .

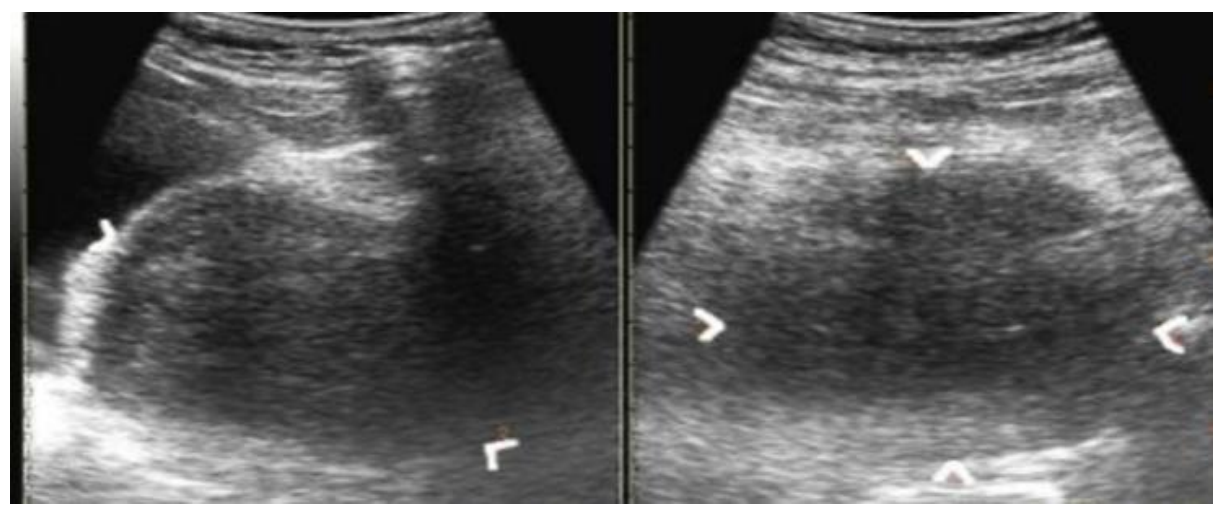

Figure (3): Pelvic ultrasound revealed markedly enlarged prostate (volume is $75 \mathrm{cc}$ ), homogeneous echopattern, grade III intra-vesical protrusion of middle lobe $(20 \mathrm{~mm})$. 


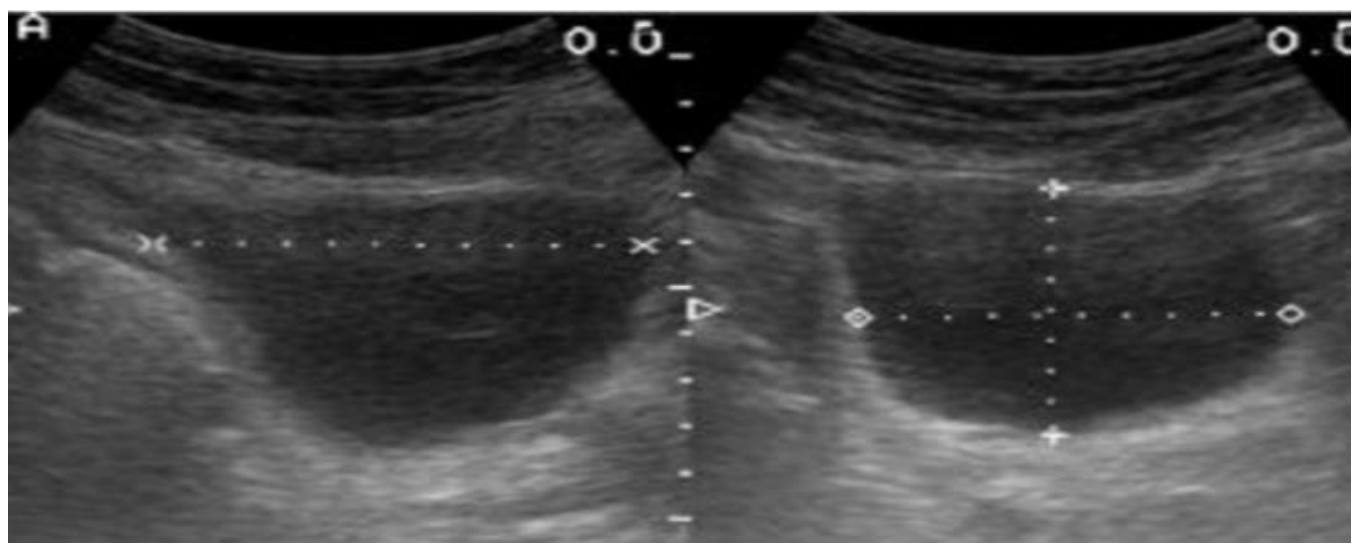

Figure (4): Pelvic ultrasound, post-voiding scan revealed PVR: $(220 \mathrm{cc})$ with diffuse thickening of mucosal lining (6 $\mathrm{mm}) \&$ fine internal echoes inside

\section{Discussion}

Obstructive uropathy can be diagnosed urgently by bedside ultrasound to realize the etiology of obstruction. Most obstruction can be corrected, but a delay in therapy can lead to irreversible renal damage. With chronic progressive obstructive uropathy, renal dysfunction may be partially or completely irreversible (4).

Urethral catheterization to relieve obstruction by enlarged prostate, antibiotics $\&$ supportive treatment, is used. If recurrence occurs more than 3 times, surgical prostatectomy must be considered. (5)

\section{Conclusion}

Bedside ultrasound is a useful tool in scanning the upper \& lower urinary tract \& to realize the etiology \& the level of urinary tract obstruction.

\section{References}

1. Kumar, Vinay, Fausto, Nelson, Fausto \& Nelso et. al.: Robbins \& Cotran Pathologic Basis of Disease $\left(7^{\text {th }}\right.$ ed). Philadelphia, Pa. (2005): Elsevier Saunders.p.1012. ISBN 978-0-7216-0187-8.

2.Walkins S, Bowra J, Sharma P, Holdgate A, Giles A \& Campbell L et. al.: Validation of emergency physician ultrasound in diagnosing hydronephrosis in ureteric colic. Emerg Med Australas. (2007) Jun. 19 (3): 188-95 (Medline).

3.Kartal M, Eray O, Erdogrn $\mathrm{T}$ \& Yilmaz S: Prospective validation of a current algorithm including bedside ultrasound performed by emergency physicians for patients with acute flank pain suspected for renal colic. Emerg Med J. (2006) May 13 (5): 341-4 (Medline).

4.Dickman E, Tessaro MO, Arroyo AC, Haines LE \& Marshall JP: Clinician-performed abdominal 
ultrasonography. Eur J Trauma Emerg Surg (2015), 41 (5): 481-92 (Medline).

5. Hassani SA, Fathi M, Daadpey M, Zare MA, emergency physician performed acute abdominal pain: A prospective study. Clin Imag. (2015) MayJun 39 (3): 476-9 (Medline).

To cite this article: Ghada M. Abdel-Rafee, Hassan Aboul-Enein. Infra-vesical Obstructive Uropathy Secondary to Benign Prostatic Enlargement. BMFJ 2020; 37 (surgical issue); 76-80. DOI: $10.21608 /$ bmfj.2020.29243.1256 